\title{
Parasite-induced surfacing in the cockle Austrovenus stuchburyi: adaptation or not?
}

\author{
D. M. TOMPKINS, K. N. MOURITSEN \& R. POULIN \\ Department of Zoology, University of Otago, Dunedin, New Zealand
}

\author{
Keywords: \\ complex life cycle; \\ Curtuteria australis; \\ extended phenotype; \\ oystercatcher; \\ parasite manipulation; \\ simulation model; \\ trematode.
}

\begin{abstract}
Parasite manipulation of host behaviour is a compelling example of the extended phenotype. However, in many cases, such manipulation may be incorrectly assumed. Previous work has demonstrated that Austrovenus stuchburyi cockles stranded on mud-flat surfaces due to an inability to re-burrow both contain significantly more metacercariae of the trematode Curtuteria australis and are predated by the definitive host of this parasite at a faster rate than burrowed cockles. These results have been interpreted as strong evidence for a manipulation of cockle behaviour by the trematode to facilitate transmission to the definitive host. The model presented here, however, indicates that the selective advantage to the parasite of the altered host behaviour is currently of a negligible level at our study site that is highly unlikely to have been realized as an adaptation over evolutionary time. Hence, there are no grounds on which the more parsimonious explanation, that the altered host behaviour observed is simply an incidental side-effect of infection, can be rejected. We thus maintain that for any change in the behaviour of infected hosts to be confirmed as potentially a parasite trait that has evolved in response to selection, the adaptive benefit taking into account the entire parasite life cycle may need to be considered.
\end{abstract}

\section{Introduction}

The adaptive benefit of many traits is widely accepted without argument, with the frequent belief that traits showing a design that appears perfectly suited to their function cannot be accidents of evolution. However, the potential costs associated with these traits, and the fact that they may simply be the fortuitous outcomes of evolutionary constraints, are often ignored (Gould \& Lewontin, 1979). The ability of parasites to alter the behaviour, colouration or morphology of their hosts in ways that facilitate their own transmission is one such suite of traits, also providing compelling evidence for the extended phenotype (i.e. the genes of one organism having visible effects in another organism, with fitness benefits reaped by the former; Dawkins, 1982). Indeed,

Correspondence: Daniel M. Tompkins, Department of Zoology, University of Otago, PO Box 56, Dunedin, New Zealand.

Tel.: (64) 3479 5848; fax: (64) 3479 7584;

e-mail: daniel.tompkins@stonebow.otago.ac.nz some examples of what parasites do to their hosts are quite remarkable (see Moore, 2002 for review), and therefore unlikely to be mere pathological side-effects of infection. However, a few critics have warned that a documented change in host behaviour that can be argued to benefit the parasite, unless it involves a complex mechanism with clear signs of purposive design, cannot be taken as evidence for adaptation without quantitative assessment of its overall effect on parasite fitness (Moore \& Gotelli, 1990; Poulin, 1995, 2000). Without such an assessment, the more parsimonious null hypothesis that altered host behaviour is simply an incidental (if fortuitous) side-effect of infection cannot be rejected. Although studies have emphasized the importance of considering biological complexity and functionality when assessing potential parasite adaptations (see, for example, Brown et al., 2001), none have explored the fitness gain to the parasite of an inferred manipulation in the context of its entire life cycle through the application of simulation modelling. We would argue, however, that such an 
approach may be essential when testing against the nonadaptive null hypothesis, since even a manipulation of great benefit at one stage of the life cyle may mean little in terms of overall parasite fitness if transmission success at that stage is not a limiting factor.

Here, we use this novel approach to critically assess a host/parasite system for which there is compelling evidence for parasite manipulation of host behaviour, to determine whether estimates of the selective advantage of the altered behaviour are of a magnitude that make it likely to have been selected for as a parasite adaptation over evolutionary time. The trematode Curtuteria australis (Echinostomatidae) cycles through three hosts in the New Zealand intertidal zone (Allison, 1979). Miracidial larvae hatched from eggs infect the whelk Cominella glandiformis, in which they multiply asexually to produce cercariae, the next free-swimming infective stage. Cercariae then enter the cockle Austrovenus stutchburyi via the filtration current and encyst as metacercariae in the bivalve's foot. The life cycle is completed when infected cockles are ingested by oystercatchers, Haematopus spp., the definitive hosts in which adult flukes live. It is at the cockle-to-bird stage of the cycle that host manipulation by the parasite has been inferred. Normal, healthy cockles live burrowed $1-3 \mathrm{~cm}$ under the intertidal zone sediment surface. However, as a cockle accumulates metacercariae in its foot, its ability to burrow decreases until the cockle is incapable of re-burrowing when accidentally exposed at the sediment surface (Thomas \& Poulin, 1998; Mouritsen, 2002). The accumulating metacercariae not only replace muscle tissue, but also directly interfere with the foot's mechanical performance (Mouritsen, 2002). Two independent predation tests have revealed that cockles exposed at the surface are between five and seven times more vulnerable to predation by oystercatchers than burrowed cockles (Thomas \& Poulin, 1998; unpublished data) and thus the inhibition of the cockle's burrowing ability apparently increases the transmission rate of the parasite to its definitive host. However, as the inferred parasite adaptation is neither complex nor strongly evident of a purposive design, we would argue that a test against the nonadaptive null hypothesis is required to justify the altered host behaviour being considered a parasite manipulation.

One reason why the observed change in host behaviour may not be adaptive is that there are potentially associated costs, as well as benefits, to the parasite. In particular, the inability of infected cockles to burrow may increase rates of ingestion of parasite metacercariae by non-host predators in two ways. First, cockles exposed at the sediment surface are also more vulnerable to predation by whelks (Mouritsen $\&$ Poulin, 2003a). Although the metacercariae can survive in whelks for a few days and may yet end up in birds if the latter prey on whelks, whelk predation results in major losses of metacercariae (McFarland et al., 2003).
Secondly, cockles at the sediment surface face a high risk of having their foot cropped by the fish Notolabrus celidotus (Mouritsen \& Poulin, 2003b). Thus, on average, a metacercariae in the foot of a surfaced cockle is about 500 times more likely to be ingested by a fish than one in a burrowed cockle (see Table 2). Hence, it is possible that any fitness gain to the parasite through an increase in the transmission rate of parasite stages from the intermediate to the definitive host may be negated by the increased loss of infective stages to these two non-host predators. Although some parasites appear to alter intermediate host behaviour not only to increase transmission to definitive hosts, but also to minimize losses to non-host organisms (e.g. Levri, 1998), the observed effect of the parasite would not allow such flexibility - either a cockle is able to burrow or it is not. Thus, losses of metacercariae to non-host organisms (and the costs to parasite fitness that they reflect) are an integral part of the model constructed, which simulates both the flow of parasite stages and the number of infected hosts in this complex system, and the magnitude of the parasite 'manipulation' of cockle behaviour at a tidal stretch of mud-flats located on the Otago Peninsular, New Zealand. This location (Company Bay) was chosen as the mean metacercariae burden of cockles at this site (and likewise the population scale impact of the parasite) is at the high end of the observed scale. Thus, we assumed that failure to reject the null hypothesis using the best parameter estimates from this site would be strong evidence for a lack of current adaptive benefit from the inferred manipulation.

\section{Methods}

\section{Model formulation}

The life cycle of $C$. australis was modelled using a linked set of differential equations, simulating (on a daily basis) the accumulation of live $(L)$ and dead $(D)$ metacercariae in cockles, the survival of metacercariae $(T)$ in the gut of whelks acting as temporary transport hosts, the population of flukes $(H)$ in the definitive host, and the number of infected whelks (I) present in the system:

$$
\begin{gathered}
\frac{d L}{d t}=\beta I \lambda_{I}-P_{L B}-P_{L W}-P_{L F}-\left(\alpha_{C}+\alpha_{L}\right) L \\
\frac{d D}{d t}=\alpha_{L} L-P_{D B}-P_{D W}-P_{D F}-\left(\alpha_{C}+\alpha_{D}\right) D \\
\frac{d T}{d t}=\left(1-\alpha_{M}\right) P_{L W}-\left(\alpha_{T}+\mu_{I B}\right) T \\
\frac{d H}{d t}=e^{-a H}\left(P_{L B}+\mu_{I B} T\right)-\alpha_{H} H
\end{gathered}
$$




$$
\frac{d I}{d t}=e^{-a H} \beta \frac{N_{W}}{N_{C}} H \lambda_{H}-\left(\alpha_{I}+\mu_{I B}\right) I
$$

In eqn (1), $\beta$ is the transmission rate (to cockles) of cercariae produced by infected whelks. The same term appears in eqn (5) for the transmission rate (to whelks) of miracidia hatched from eggs defecated by the definitive host, although here it is adjusted to take into account the different abundances of whelks and cockles in Company Bay $\left(N_{W} / N_{C}\right)$. This formulation assumes that the transmission rate per host individual is the same for both cercariae and miracidia. This is a logical assumption to make as both forms of infective stages are being transmitted at the same locality in the same medium. Note that any cercariae and miracidia that fail to infect either cockles or whelks respectively on the day they are produced are assumed to be lost from the system. This is realistic as a result of the tidal nature of Company Bay. The time delay between egg defecation and miracidial emergence from eggs (c. 10 days; Allison, 1979) and the time delay between miracidial penetration of whelks and cercariae production (c. 3 weeks; Allison, 1979) are not included in the model as they do not significantly affect the equilibrium dynamics.

The $\lambda_{I}$ [eqn (1)] and $\lambda_{H}$ [eqn (5)] denote the production rates of cercariae and eggs, by infected whelks and flukes in the definitive host respectively. $\alpha_{C}$ [eqns (1) and (2)], $\alpha_{L}$ [eqn (1)], $\alpha_{H}$ [eqn (4)] and $\alpha_{I}$ [eqn (5)] are the mortality rates of cockles, metacercariae, flukes and infected whelks, respectively, whereas $\alpha_{D}$ [eqn (2)] and $\alpha_{T}$ [eqn (3)] are the clearance rates of dead metacercariae from the cockle foot and live metacercariae from the whelk gut, respectively, and $\alpha_{M}$ [eqn (3)] is the metacercariae mortality that occurs initially upon ingestion by whelks. Whelk mortality is ignored in eqn (3) as this is an insignificant route of metacercariae loss compared with the rate of clearance from the gut.

Whereas, $\mu_{I B}$ [eqns (3-5)] is the predation rate of infected whelks by birds, $P_{X Y}$ [eqn $\left.(1-4)\right]$ is the number of $X$ (either live or dead metacercariae in cockles) ingested by $Y$, with $Y=B, W$ and $F$ denoting predation by birds, whelks and fish (cropping) respectively, where:

$$
P_{X Y}=X \frac{\mu_{J Y}+S\left(1.4 \mu_{K Y}-\mu_{J Y}\right)}{1+0.4 S}
$$

in which $\mu_{J Y}$ and $\mu_{K Y}$ are the predation rates of burrowed and surfaced cockles respectively by $Y$, and $S$ is the proportion of the cockle population that is surfaced, being dependent on the intensity of metacercariae infection in the following manner:

$$
\begin{array}{r}
S=\frac{0.0000366(L+D)}{N_{C}}-0.0001047 \\
\quad\left[\text { if }(L+D) / N_{C} \geq 2.86, \text { else } S=0\right]
\end{array}
$$

These equations are based on the observation that the intensity of metacercariae infection in surfaced cockles is c. 1.4 times that in burrowed cockles (after controlling for
Table 1 Intensity of infection (mean \pm SE), by metacercariae of the trematode Curtuteria australis, in surfaced vs. burrowed cockles from three sites in Company Bay, New Zealand (unpublished data).

\begin{tabular}{lllllll}
\hline & \multicolumn{2}{l}{ Intensity observed $(n)$} & & \multicolumn{2}{l}{ Adjusted for cropping } & \\
\cline { 2 - 3 } Site & Surfaced & Burrowed & & Surfaced & Burrowed & $\begin{array}{l}\text { Surfaced/ } \\
\text { burrowed }\end{array}$ \\
\hline 1 & $140 \pm 15(72)$ & $118 \pm 15(73)$ & 163 & 118 & 1.38 \\
2 & $395 \pm 38(35)$ & $322 \pm 35(36)$ & 460 & 322 & 1.43 \\
3 & $568 \pm 46(74)$ & $457 \pm 35(79)$ & 661 & 457 & 1.45 \\
\hline
\end{tabular}

The mean values adjusted for the different rates of foot cropping by fish for surfaced vs. burrowed cockles taken into account an average loss of metacercariae from surfaced and burrowed cockles of $14.18 \%$ and $0.09 \%$ respectively (see Methods).

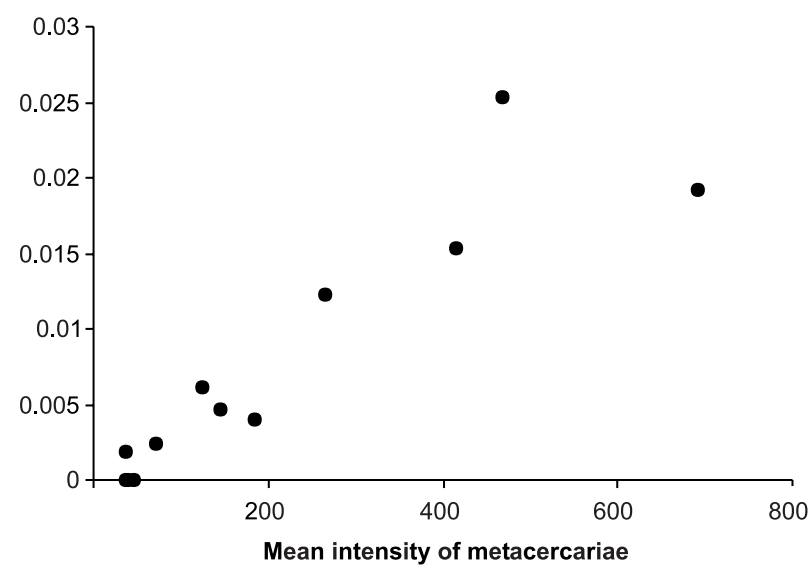

Fig. 1 Relationship between the mean intensity of infection by metacercariae of the trematode Curtuteria australis, and the proportion of cockles that are surfaced in Company Bay, New Zealand ( $n=12$ sites). Data obtained from Mouritsen \& Poulin (2003a).

the different rates of foot cropping by fish for surfaced vs. burrowed cockles - see below), irrespective of global intensity (Table 1), and the previously documented positive relationship between the intensity of metacercariae infection and the proportion of cockles that are found on the surface (Fig. 1).

Density dependence in the model is in the form of reduced establishment success (from 100\%) of ingested metacercariae and reduced fecundity of flukes in the definitive host with increasing fluke population size $\left[e^{-a H}\right.$ in eqns (4) and (5)]. As we have no specific information on the population dynamics of $C$. australis infections in birds, the magnitude of the density dependence effect ( $a$ ), by default, is assumed to be the same for both establishment success and fecundity. Based on experimental infections in the model Echinostoma revolutum/chicken system (with E. revolutum and $C$. australis being in the same taxonomic family, the Echinostomatidae), this is a reasonable assumption (Fried, 1984; Fried \& Freeborne, 1984). As the proportion of Curtuteria-infected whelks in 


\begin{tabular}{lll}
\hline Parameter & Symbol & Value \\
\hline Number of cockles in Company Bay & $N_{C}$ & 3103200 \\
Number of whelks in Company Bay & $N_{W}$ & 172800 \\
Cercariae emergence from infected whelks & $\lambda_{I}$ & 100 \\
Egg production by flukes in the definitive host & $\lambda_{H}$ & 19.58 \\
Cockle mortality & $\alpha_{C}$ & 0.000274 \\
Metacercariae mortality & $\alpha_{L}$ & 0.00184 \\
Fluke mortality & $\alpha_{H}$ & 0.026 \\
Infected whelk mortality & $\alpha_{I}$ & 0.000548 \\
Dead metacercariae clearance from the cockle foot & $\alpha_{D}$ & 0.00274 \\
Live metacercariae clearance from the whelk gut & $\alpha_{T}$ & 0.333 \\
Metacercariae mortality upon ingestion by whelks & $\alpha_{M}$ & 0.194 \\
Predation of infected whelks by birds & $\mu_{I B}$ & 0.000122 \\
Predation of burrowed cockles by birds & $\mu_{J B}$ & 0.000125 \\
Predation of surfaced cockles by birds & $\mu_{K B}$ & 0.00064 \\
Predation of burrowed cockles by whelks & $\mu_{J W}$ & 0.000165 \\
Predation of surfaced cockles by whelks & $\mu_{K W}$ & 0.000965 \\
Cropping of metacercariae from burrowed cockles & $\mu_{J F}$ & 0.000000533 \\
Cropping of metacercariae from surfaced cockles & $\mu_{K F}$ & 0.000266 \\
Cercariae transmission to cockles & $\beta$ & $?\left(N_{W} / N_{C}\right)$ \\
Miracidia transmission to whelks & $a$ & $?$ \\
Fluke density dependence in the definitive host & & $?$ \\
\hline
\end{tabular}

Table 2 Parameter values for the simulated life cycle of the trematode Curtuteria australis infecting whelks, cockles and birds in Company Bay, New Zealand.

All values (bar $N_{C}, N_{W}$ and $a$ ) are expressed as daily rates.

Company Bay is, on average, only c. 5\% (see below), density dependence is unlikely to affect the infection rate of uninfected whelks by miracidia. Also, to our knowledge, there is no evidence suggesting density dependence in the rate at which metacercariae accumulate in cockles; even in the most heavily infected specimens from Company Bay, metacercariae occupy only about $25 \%$ of the cockle foot (unpublished data), indicating that there is plenty of space left for additional infections.

The model is illustrated, in flow diagram form, in Fig. 2. Note that the model contains both macroparasite and microparasite elements, with eqns (1-4) simulating parasite numbers (in cockles, birds, and the gut of whelks), whereas eqn (5) simulates infected host individuals (whelk 'cercarial factories'). Whereas the cockle and whelk populations of Company Bay are assumed to remain constant at $N_{C}$ and $N_{W}$ respectively, with densitydependent recruitment compensating for all mortality rates, the size of the bird population is not considered (rather the fluke population infecting all bird hosts at Company Bay is simulated). Also note that the documented relationship between cockle size, parasite intensity, and oystercatcher foraging preference (Norris, 1999) is not included in the model as, although there is a significant positive relationship between cockle shell length and metacercariae intensity in the system studied, it explains very little of the variance observed $\left(r^{2}<0.08\right.$; Poulin et al., 2000). Furthermore, even if definitive hosts could avoid large numbers of parasites in this system by selective foraging, this would only act to reduce the selective advantage of the inferred manipulation, making rejection of the nonadaptive null hypothesis less likely.

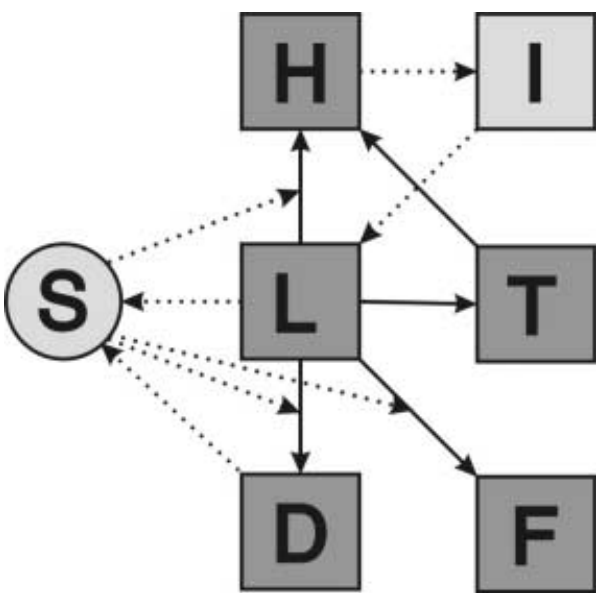

Fig. 2 Flow diagram for the simulated life cycle of the trematode Curtuteria australis infecting whelks, cockles and birds in Company Bay, New Zealand. Dark boxes denote accumulations and/or losses of parasite stages (the macroparasite part of the model) with, as in the model equations, $L, D, T, H$ and $I$ denoting live metacercariae in cockles, dead metacercariae in cockles, live metacercariae in the whelk transport host, flukes in the definitive host, and infected whelks respectively. $F$ denotes the loss of metacercariae to fish via cropping of the cockle foot. The light box, $I$, denotes the number of infected whelks (the microparasite part of the model), and the circle denotes the variable $S$ (the proportion of the cockle population that is surfaced). Solid arrows indicate the flow of macroparasite stages, whereas dotted arrows indicate influences.

Recently, a second species of trematode was found encysted in the foot of cockles, along with $C$. australis (unpublished data). Like C. australis, it belongs to the 
family Echinostomatidae; it uses a different snail first intermediate host, but shares cockles and oystercatchers, as second intermediate and definitive hosts respectively, with $C$. australis. The relative proportions of the two trematodes vary among localities in Otago Harbour, and probably fluctuate in time as well. However, they show the same preference for encysting near the tip of the foot of cockles, and they have roughly similar sizes (unpublished data). We consider them to be ecological equivalents, and have not distinguished between them in the model.

\section{Model parameterization}

Parameter estimates (daily values averaged across seasons) were obtained from the literature, including our own work, and unpublished data (see Table 2). Numbers of cockles $\left(N_{C}\right)$ and whelks $\left(N_{W}\right)$ present in Company Bay were estimated from mean densities of $431 \mathrm{~m}^{-2}$ and $24 \mathrm{~m}^{-2}$ respectively and an approximate area of available habitat of $7200 \mathrm{~m}^{2}$ (unpublished data). The rates of predation of cockles by birds $\left(\mu_{J B}\right.$ and $\mu_{K B}$ for burrowed and surfaced cockles respectively) and whelks ( $\mu_{J W}$ and $\mu_{K W}$ for burrowed and surfaced cockles respectively) were estimated as half that observed during the yearly peak period of predation and expressed in terms of the proportion of the standing stock of burrowed and surfaced cockles taken per day (unpublished data). Cropping rates of burrowed and surfaced cockles by fish ( $\mu_{J F}$ and $\mu_{K F}$ respectively) were calculated as those required to maintain equilibrium levels of $13.9 \%$ burrowed and $82.9 \%$ surfaced cockles cropped, where cropped burrowed and surfaced cockles have lost, on average, $0.68 \%$ and $17.1 \%$ of their metacercarial loads respectively (unpublished data), in the face of cockle recruitment (of uncropped individuals) replacing the losses to the cockle population caused by natural mortality $\left(\alpha_{C}\right)$, bird predation $\left(\mu_{J B}\right.$ and $\mu_{K B}$ for burrowed and surfaced cockles respectively) and whelk predation $\left(\mu_{J W}\right.$ and $\mu_{K W}$ for burrowed and surfaced cockles respectively). The predation rate of infected whelks by the definitive host $\left(\mu_{I B}\right)$ was calculated based on the assumption that whelks make up 5\% of the birds' diet, with the remaining $95 \%$ of the diet made up by cockles (Baker, 1974). According to our parameter values, with c. $0.5 \%$ of the cockle population of Company Bay occurring on the surface of the mud-flats (see below), an average of 396 individual cockles are taken by birds from Company Bay each day. Thus, the whelk proportion of the bird diet consists of an estimated 21 individual whelks per day.

Both the clearance rate of live metacercariae from the whelk gut $\left(\alpha_{T}\right)$ and the metacercariae mortality that occurs initially upon ingestion by whelks $\left(\alpha_{M}\right)$ were calculated from experimental infections (McFarland et al., 2003), whilst the clearance rate of dead metacercariae from the cockle foot $\left(\alpha_{D}\right)$ was estimated from unpublished data. The natural mortality rate of cockles
$\left(\alpha_{C}\right)$ was estimated from an observed maximum lifespan of $c .20$ years (unpublished data). The mortality rate of metacercariae in cockles $\left(\alpha_{L}\right)$ was assumed to be similar to values reported for other echinostomes (Donges, $1969)$, and the mortality rate of infected whelks $\left(\alpha_{I}\right)$ was deduced from Huxham et al. (1993). The mortality rate of flukes in the definitive host $\left(\alpha_{H}\right)$ was calculated using the body size regression approach of Trouvé et al. (1998) (with an average body size for C. australis of $1.8 \mathrm{~mm}$; Allison, 1979), plus a small amount to account for fluke loss because of host mortality. This same approach was used to give an estimate for fluke fecundity in the definitive host of 23.5 eggs per day. Since, of the 39 day lifespan, C. australis flukes are estimated as being prepatent for 6.5 days (again using the body size regression approach), the fecundity value was adjusted to give an average rate per day $\left(\lambda_{H}\right)$ across the entire lifespan. The rate of cercarial emergence from infected whelks $\left(\lambda_{I}\right)$ was estimated from the experimental values recorded for C. australis in Allison (1979).

\section{Model simulation}

Model properties were explored by numerical simulation. Values for either the transmission rate $(\beta)$ of cercariae to cockles (and likewise the transmission rate of miracidia to whelks) or the strength of density dependence influencing flukes in the definitive host $(a)$ are not available. Hence, model behaviour was analysed to ascertain the al $\beta$ parameter region in which the model output conformed to observed characteristics of the parasite system in Company Bay - a mean intensity of 150 metacercariae (both live and dead) per cockle, and an average proportion of infected whelks (producing cercariae) in the whelk population of $5 \%$ (unpublished data). Only one combination of $a$ and $\beta$ satisfied the above requirements (see Results) and these were thus the parameter estimates used in subsequent simulations.

The selective potential of the surfacing behaviour in the cockles as a parasite-induced manipulation was investigated by comparing parasite population equilibria for the model constructed including the surfacing behaviour as a manipulation to a version of the model with the surfacing behaviour removed (i.e. the proportion of the cockle population surfaced, $S$, maintained at $0)$. The selective advantage(s) of a parasite that induces the surfacing behaviour over one that does not, was calculated as the increase in the equilibrium fluke population size attained over that of a non-surfacing parasite, divided by the non-surfacing equilibrium. This is the correct point at which to estimate parasite fitness, as it is in the definitive host that competition between parasites occurs (see model formulation above). A more complex version of the model constructed, which placed surfacing and non-surfacing parasites together in the same system, verified that any selective advantage of the induced behaviour would lead to the loss of the 
non-surfacing parasite at a rate proportional to the magnitude of ' $\mathrm{s}$ '.

The selective advantage of the parasite-induced surfacing behaviour in cockles was assessed both in the presence (as in the model constructed) and absence of whelk predation of cockles and cropping of the cockle foot by fish, as these trophic relationships may have evolved after the potential parasite manipulation (only being selected for once the parasite causing the hosts to surface became established in the host population, resulting in a constant supply of exposed cockles). It thus may be argued that even if there is currently no recognizable selective advantage to the parasite of the inferred manipulation, the behaviour may have evolved at a time when the ecological conditions were different, metacercariae in surfaced cockles were not lost to non-host predators, and the costs associated with adopting the behaviour were lower. Likewise, the host/parasite association may also have evolved under different levels of predation of burrowed and surfaced cockles by the definitive oystercatcher hosts than have been estimated from Company Bay. Sensitivity analyses were thus conducted to determine the robustness of the estimated selective advantage of the inferred manipulation in the face of changes in the model parameters $\mu_{J B}$ and $\mu_{K B}$. Such analyses were also conducted on the unknown parameters $a$ and $\beta$.

\section{Results}

Model equilibria of a mean intensity of 150 metacercariae per cockle, and an average proportion of infected whelks in the whelk population of $5 \%$ were only obtained at the a/ $\beta$ parameter combination of $a=0.002015$ and $\beta=0.8373$. These values were thus used to parameterise the model for all subsequent simulations. At these parameter values an equilibrium fluke population size in the definitive host of $H=3068$ was observed, with $0.54 \%$ of the cockle population being surfaced and a ratio of live : dead metacercariae in the cockle foot of $1.8: 1$. Interestingly, these equilibrium values barely alter if metacercariae transmission to birds via ingested whelks is not included in the model (mean intensity per cockle $=150$, proportion of infected whelks $=5 \%$, fluke population size $=3068$ ). This confirms the view that although metacercariae ingested by whelks during predation on cockles can remain viable, with the potential to infect the definitive oystercatcher host if such 'transport' whelks are themselves predated upon, this route of infection is of little significance in the parasite life cycle (McFarland et al., 2003). This is primarily due to the relatively fast clearance rate of whelk gut contents (taking c. 3 days; McFarland et al., 2003), and the relatively low estimated rate of whelk predation by the definitive host (Table 2).

When the model was run with the surfacing behaviour removed, only minor changes in parasite equilibria were observed, regardless of whether whelk predation of cockles and cropping of the cockle foot by fish were included or not (Table 3). In terms of the selective advantage of a parasite that induces the surfacing behaviour over one that does not, $s=0.0021$ in the presence of non-host predators, and $s=0.0026$ in their absence. At this order of magnitude, it is highly unlikely that a mutation in the parasite population giving rise to the induced behaviour would be sufficiently advantageous for its fixation in the face of genetic drift - i.e. the advantage of surfacing is unlikely to be realized in a natural population as a parasite manipulation of host behaviour (Levinton, 2001). For example, assuming $100 \%$ heritability, the probability of fixation for such a mutation occurring at an initial frequency of 0.001 (i.e. 1 in 1000 parasites induces the behaviour) in a population of 10000 flukes is calculated as being $<5 \%$ (Ewens, 1979). Thus, even accounting for the fact that the parasites in Company Bay (equilibrium of just over 3000 flukes - see Table 3) may actually be part of a larger population (with fixation more likely in larger populations), we are unable to reject the null hypothesis that the surfacing behaviour is simply a side-effect of infection.

The sensitivity analyses conducted show that this result varies little with changes in the values of the unknown parameters $a$ and $\beta$ (Fig. 3). Maximizing the rate of cercarial transmission $(\beta)$ does little to increase the selective advantage of the behaviour, whilst the

\begin{tabular}{|c|c|c|c|c|}
\hline & \multicolumn{2}{|c|}{$\begin{array}{l}\text { Predation and } \\
\text { cropping included }\end{array}$} & \multicolumn{2}{|c|}{$\begin{array}{l}\text { Predation and } \\
\text { cropping excluded }\end{array}$} \\
\hline & S & NS & S & NS \\
\hline Mean intensity of metacercariae infection in cockles & 150 & 153 & 160 & 162 \\
\hline Ratio of live : dead metacercariae in the cockle foot & $1.8: 1$ & $1.8: 1$ & $1.71: 1$ & $1.71: 1$ \\
\hline Percentage of whelk population infected & 5.0 & 5.1 & 4.8 & 4.9 \\
\hline Fluke population size in the definitive host & 3068 & 3062 & 3087 & 3079 \\
\hline Percentage of cockle population surfaced & 0.54 & NA & 0.57 & NA \\
\hline
\end{tabular}

Table 3 Equilibrium values for the simulated life cycle of the trematode Curtuteria australis infecting whelks, cockles and birds in Company Bay, New Zealand, for the model constructed containing the surfacing behaviour of cockles as a manipulation (S) vs. the version of the model with the surfacing behaviour removed (NS).

This comparison was conducted in both the presence (as in the model constructed) and absence of whelk predation of cockles and cropping of the cockle foot by fish. 
(a)

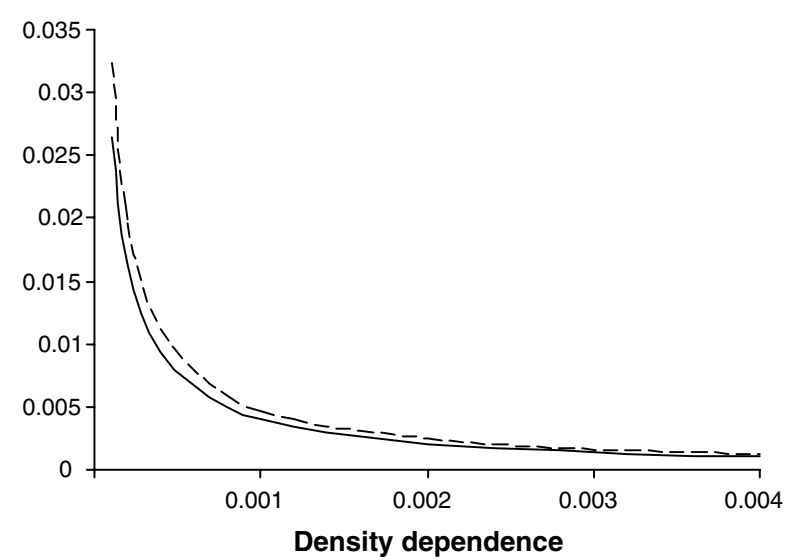

(b)

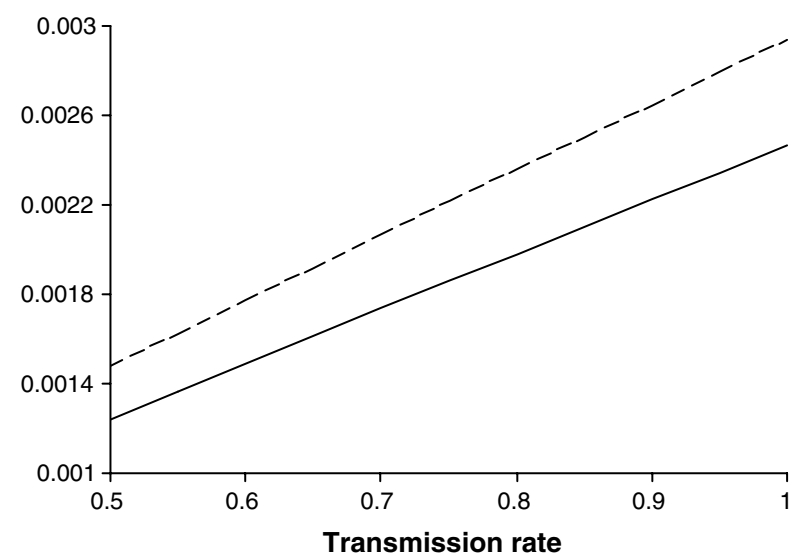

Fig. 3 Sensitivity analysis demonstrating how the selective advantage of the inferred parasite adaptation to manipulate the behaviour of the cockle intermediate host varies with changes in the unknown parameters (a) fluke density dependence in the definitive host, and (b) the transmission rate of cercariae to cockles (and likewise the transmission rate of miracidia to whelks), in models both with (solid lines) and without (dashed lines) cockle predation by non-host organisms. Selection coefficients were calculated from equilibrium fluke population sizes generated by model runs with and without the inferred manipulation.

strength of density dependence in the definitive host (a) has to drop to 0.0008 before the probability of fixation in the above calculation increases above $10 \%$ (at a selective advantage of $s=0.0055$ ). At this level parasite intensity in the whelks, cockles and birds is already predicted by the model to be unrealistically high (c. $12.5 \%$ of the whelk population infected, 370 metacercariae per cockle, and a population of 8000 flukes).

Altering the values for the predation rates of cockles by the definitive bird host had more varied results (Fig. 4). The predicted selective advantage of the surfacing behaviour, in either the presence or absence of non-host predators, again altered little with changes in the (a)

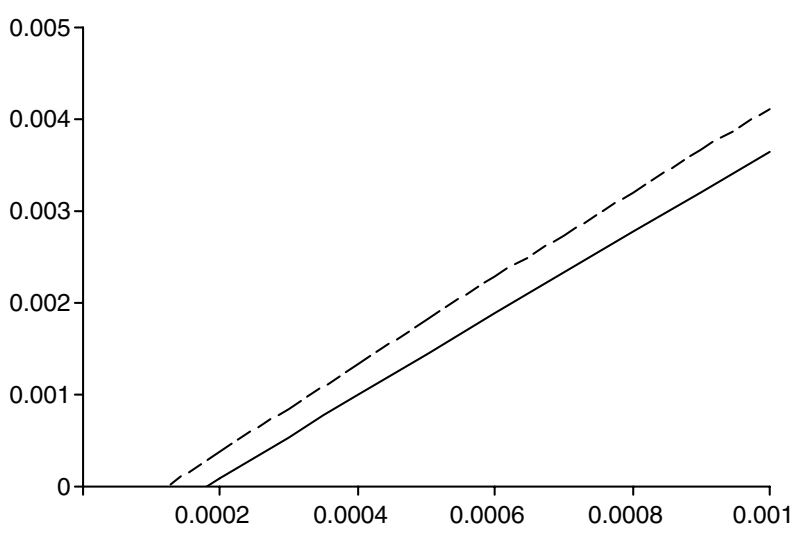

(b)

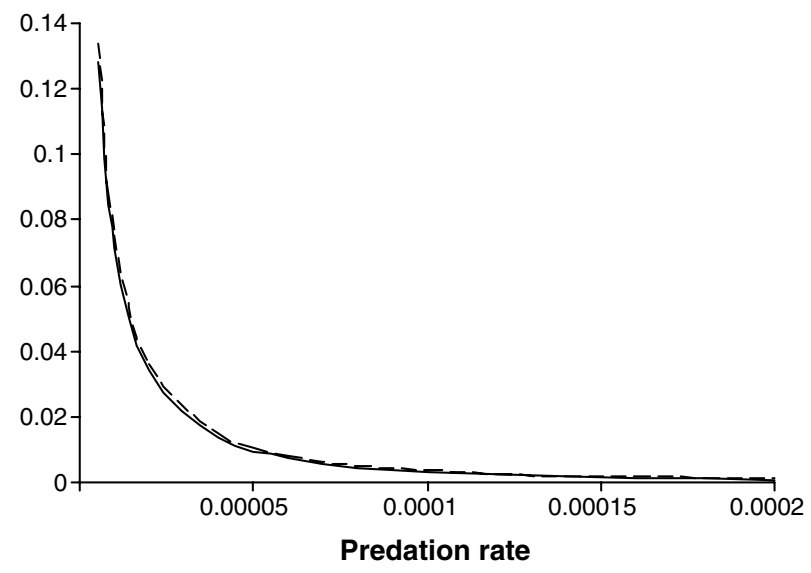

Fig. 4 Sensitivity analysis demonstrating how the selective advantage of the inferred parasite adaptation to manipulate the behaviour of the cockle intermediate host varies with changes in the predation rate of (a) surfaced cockles by birds, and (b) burrowed cockles by birds, in models both with (solid lines) and without (dashed lines) cockle predation by non-host organisms. Selection coefficients were calculated from equilibrium fluke population sizes generated by model runs with and without the inferred manipulation.

predation rate of surfaced cockles by oystercatchers. It was greatly affected, however, by changes in the predation rate of burrowed cockles by oystercatchers with a decrease in the predation rate of $40 \%$ (to 0.00007 ) increasing the calculated fixation probability of the induced behaviour as an adaptation to $10 \%$, and a decrease of $80 \%$ (to 0.000025 ) increasing it to $50 \%$. Interestingly, at such a level $(50 \%$ probability of fixation), the parasite intensities predicted by the model do not alter unreasonably - the proportion of the whelk population infected increases to $8.5 \%$, the number of metacercariae per cockle increases to 270 (with the proportion of the cockle population surfaced at $1 \%$ ), and the size of the fluke population in the definitive host decreases to 2750 . 


\section{Discussion}

In this study, we took the novel approach of simulating a complex parasite life cycle to investigate the adaptive potential of an inferred parasite manipulation of host behaviour. We would argue that such an approach, designed to quantify overall effects on parasite fitness, is a useful test of whether documented changes in host behaviour are to be invoked as being beneficial to the fitness of an infecting parasite, and should be applied in more studies. As the scientific method highlights, one should always maintain the most parsimonious explanation as one's null hypothesis (altered host behaviour being simply an incidental side-effect of infection in this case), and the burden of proof should be on demonstrating otherwise (that the altered behaviour is indeed a parasite adaptation). Too many times this approach is not taken in studies of parasite manipulation of host behaviour, with manipulation often being accepted precociously.

All evidence suggests that the model constructed accurately captures the dynamics of the parasite/host system in Company Bay under investigation. Although two crucial parameters (the transmission rate of cercariae to cockles and the strength of density dependence influencing flukes in the definitive host) had to be estimated, model simulations run with the determined values not only fitted the selection criteria for the estimation procedure, but also resulted in all other aspects of the equilibrium model being structured in agreement with the natural system. For example, at equilibrium for all simulations conducted, the ratio of live : dead metacercariae in the cockle foot deviated little from 1.8 : 1 (Table 3 ). The resulting proportion of dead metacercariae $(35.7 \%)$ is similar to that observed around the Otago Peninsula (mean $\pm \mathrm{SD}, 0.281 \pm 0.153 ; n=80$; unpublished data), as is the proportion of the cockle population that is surfaced (0.54\%; see Fig. 1).

As another example, the equilibrium population size of flukes in definitive hosts at Company Bay, driving all other components of the parasite life cycle, varies little from a total of 3068 under all simulations conducted at an estimated cercarial transmission and fluke density dependence parameter values. Since the number of oystercatchers utilizing Company Bay over the course of the year has been observed at approximately four individuals per day (unpublished data), this gives a mean intensity of flukes in the definitive host of 767 parasites. This is well within the range of mean intensities reported for oystercatcher gut helminth infections (e.g. Goater et al., 1995). Furthermore, the numbers of intermediate hosts estimated as being predated by definitive hosts at these equilibrium levels (a mean of 104 prey items per bird per day) gives an intake rate similar to that recorded for oystercatchers predating mainly on cockles in others studies (e.g. Norris \& Johnstone, 1998).

The fit of the model to characteristics of the natural parasite/host system being studied adds confidence to the conclusion that the benefit to the parasite incurred from the change in intermediate host behaviour caused by metacercarial accumulation in the cockle foot is negligible in Company Bay. That the parasite has the ability to impair the burrowing or crawling activity of its host is not in question, as is the case with other burrowing bivalve/ trematode parasite systems (Mouritsen, 1997; Desclaux et al., 2002), as the direct effect of $C$. australis on the burrowing behaviour of the cockle $A$. stutchburyi has been well-documented (Thomas \& Poulin, 1998; Mouritsen, 2002). Rather, it seems that the magnitude of the selective advantage that the inferred manipulation confers to the trematode is currently of a level that is unlikely to achieve fixation as a parasite adaptation - the model equilibria for numerical simulations run with and without the surfacing behaviour are almost identical (Table 3). This lack of any real adaptive potential of the inferred manipulation is not due to the increased loss of infective stages in surfaced cockles through predation by non-host whelks and fish, as was initially postulated. Rather, it is due to the increase in the proportion of the cockle population being found on the sediment surface in the intertidal zone resulting from parasite infection, and the subsequent increase in metacercariae uptake by the definitive host, having little effect on parasite fitness as transmission success at this stage is not a limiting factor.

Many authors have argued that for any behaviour of an infected host to be accepted as a parasite manipulation to increase transmission to the next host in the parasite life cycle, experiments demonstrating a significant increase in transmission caused by the behaviour are required (Dobson, 1988; Moore \& Gotelli, 1990; Poulin, 1995). This approach has been used in several studies (e.g. Moore, 1983; Lafferty \& Morris, 1996; Bakker et al., 1997; see review in Thomas et al., 1998). Our work here, however, shows clearly that for the system studied this criterion is not sufficient - although previous work has demonstrated that surfaced $A$. stuchburyi cockles do contain significantly more $C$. australis metacercariae than burrowed cockles, and that the rate of predation of surfaced cockles by the definitive host is significantly greater, here we show that at the point of the parasite life cycle at which it is occurring, the magnitude of the manipulation is not sufficient to make much difference to parasite fitness. There is therefore only a negligible selective advantage to the parasite associated with the surfacing behaviour ( $\mathrm{s}=0.0021)$ that is unlikely to be realized in the face of genetic drift (Levinton, 2001). Thus, given that we have used the best available parameter estimates for our system, we cannot reject the more parsimonious null hypothesis that the behaviour is simply an incidental side-effect of infection. This result strengthens the argument that evidence of an increase in transmission caused by a parasite-induced host behaviour (unless, as mentioned in the Introduction, it involves a complex mechanism with clear signs of purposive design) is not sufficient to invoke 
manipulation. Rather, one needs to show that any increase is of a magnitude of both biological and evolutionary significance. Simulation modelling is one way in which this can be achieved, with the additional advantage that initially important biological factors (non-host predation in this case) can be investigated and shown to be of no consequence, whilst subtler aspects of the biology can be revealed.

Although we have failed to reject the null hypothesis, this study is not proof that the induced surfacing is not a parasite manipulation of cockle behaviour. Indeed, whilst the sensitivity analyses conducted demonstrate that our conclusion is robust in the face of variations in fluke density dependence in the definitive host (Fig. 3a), the rate of cercariae transmission (Fig. 3b), and the predation rate of surfaced cockles by the definitive host (Fig. 4a), they also show that at reduced predation rates of burrowed cockles the selective advantage of the behaviour as a parasite-induced manipulation increases greatly (Fig. 4b). This is biologically intuitive - under such ecological conditions the benefit to a parasite of being in a surfaced intermediate host is greatly increased. Furthermore, the idea that the predation pressure on burrowed cockles by oystercatchers may be reduced in certain circumstances is not unreasonable. For example, in locations where more easily accessible polychaetes are very abundant, oystercatchers do switch their preference away from burrowed cockles (personal observation). We can thus postulate that the behaviour may have evolved as a parasite adaptation under such conditions, either in the past or at a different location. Spatial variation in transmission conditions, either at local or geographical scales, can create a mosaic of coevolutionary sites, where the selective advantages of parasite-induced host surfacing range from negligible to substantial (see Thompson, 1994). The modelling approach used has thus directed us to a new avenue of research - if we can find sites in which the parasite intensity in the intertidal community fits those predicted to occur when the adaptive potential of surfacing is greater, then quantifying predation at these sites would potentially allow us to reject the nonadaptive null hypothesis.

\section{Acknowledgments}

Some of this work is funded by a grant from the Marsden Fund. RP is supported by a James Cook Research Fellowship from the Royal Society of New Zealand. KNM is supported by the Danish Natural Science Research Council.

\section{References}

Allison, F.R. 1979. Life cycle of Curtuteria australis n. sp. (Digenea: Echinostomatidae: Himasthlinae), intestinal parasite of the South Island pied oystercatcher. N. Z. J. Zool. 6: $13-20$.
Baker, A.J. 1974. Prey specific feeding methods of New Zealand oystercatchers. Notornis 21: 219-233.

Bakker, T.C.M., Mazzi, D. \& Zala, S. 1997. Parasite-induced changes in behavior and color make Gammarus pulex more prone to fish predation. Ecology 78: 1098-1 104.

Brown, S.P., Loot, G., Grenfell, B.T. \& Guegan, J.F. 2001. Host manipulation by Ligula intestinalis: accident or adaptation? Parasitology 123: 519-529.

Dawkins, R. 1982. The Extended Phenotype. Oxford University Press, Oxford, UK.

Desclaux, C., de Montaudouin, X. \& Bachelet, G. 2002. Cockle emergence at the sediment surface: 'favourization' mechanism by digenean parasites? Dis. Aquat. Org. 52: 137-149.

Dobson, A.P. 1988. The population biology of parasite-induced changes in host behavior. Q. Rev. Biol. 63: 139-165.

Donges, J. 1969. Entwicklungs und lebensdauer von metacercarien. Zeit. Parasiten. 31: 340-366.

Ewens, W.J. 1979. Mathematical Population Genetics. SpringerVerlag, Berlin, Germany.

Fried, B. 1984. Infectivity, growth and development of Echinostoma revolutum (Trematoda) in the domestic duck. J. Helminthol. 58: 241-244.

Fried, B. \& Freeborne, N.E. 1984. Effects of Echinostoma revolutum (Trematoda) adults on various dimensions of the chicken intestine, and observations on worm crowding. Proc. Helminthol. Soc. Wash. 51: 297-300.

Goater, C.P., Goss-Custard, J.D. \& Kennedy, C.R. 1995. Population dynamics of two species of intestinal helminth in oystercatchers (Haematopus ostralegus). Can. J. Zool. 73: 296300.

Gould, S.J. \& Lewontin, R.C. 1979. The spandrels of San Marco and the Panglossian paradigm: a critique of the adaptationist programme. Proc. R. Soc. Lond. B 205: 581-598.

Huxham, M., Raffaelli, D. \& Pike, A. 1993. The influence of Cryptocotyle lingua (Digenea: Platyhelminthes) infections on the survival and fecundity of Littorina littorea (Gastropoda: Prosobranchia): an ecological approach. J. Exp. Mar. Biol. Ecol. 168: 223-238.

Lafferty, K.D. \& Morris, A.K. 1996. Altered behavior of parasitized killifish increases susceptibility to predation by bird final hosts. Ecology 77: 1390-1397.

Levinton, J.S. 2001. Genetics, Paleontology and Macroevolution. 2nd edn. Cambridge University Press, Cambridge.

Levri, E.P. 1998. The influence of non-host predators on parasite-induced behavioural changes in a freshwater snail. Oikos 81: 531-537.

McFarland, L.H., Mouritsen, K.N. \& Poulin, R. 2003. From first to second and back to first intermediate host: the complex transmission routes of Curtuteria australis (Digenea: Echinostomatidae). J. Parasitol. 89: 625-628.

Moore, J. 1983. Responses of an avian predator and its isopod prey to an acanthocephalan parasite. Ecology 64: 1000-1015.

Moore, J. 2002. Parasites and the Behavior of Animals. Oxford University Press, Oxford.

Moore, J. \& Gotelli, N.J. 1990. A phylogenetic perspective on the evolution of altered host behaviours: a critical look at the manipulation hypothesis. In: Parasitism and Host Behaviour (C. J. Barnard \& J. M. Behnke, eds), pp. 193-233. Taylor and Francis, London.

Mouritsen, K.N. 1997. Crawling behaviour in the bivalve Macoma balthica: the parasite manipulation hypothesis revisited. Oikos 79: 513-520. 
Mouritsen, K.N. 2002. The parasite-induced surfacing behaviour in the cockle Austrovenus stutchburyi: a test of an alternative hypothesis and identification of potential mechanisms. Parasitology 124: 521-528.

Mouritsen, K.N. \& Poulin, P. 2003a. Parasite-induced trophic facilitation exploited by a non-host predator: a manipulator's nightmare. Int. J. Parasitol. 33: 1043-1050.

Mouritsen, K.N. \& Poulin, P. 2003b. The risk of being at the top: foot-cropping in the New Zealand cockle Austrovenus stutchburyi. J. Mar. Biol. Assoc. UK 83: 497-498.

Norris, K. 1999. A trade-off between energy intake and exposure to parasites in oystercatchers feeding on a bivalve mollusc. Proc. R. Soc. Lond. B 266: 1703-1709.

Norris, K. \& Johnstone, I. 1998. The functional response of oystercatchers (Haematopus ostralegus) searching for cockles (Cerastoderma edule) by touch. J. Anim. Ecol. 67: 329-346.

Poulin, R. 1995. 'Adaptive' changes in the behaviour of parasitized animals: a critical review. Int. J. Parasitol. 25: $1371-1383$.

Poulin, R. 2000. Manipulation of host behaviour by parasites: a weakening paradigm? Proc. R. Soc. Lond. B 267: 787-792.
Poulin, R., Steeper, M.J. \& Miller, A.A. 2000. Non-random patterns of host use by the different parasite species exploiting a cockle population. Parasitology 121: 289-295.

Thomas, F. \& Poulin, R. 1998. Manipulation of a mollusc by a trophically transmitted parasite: convergent evolution or phylogenetic inheritance? Parasitology 116: 431-436.

Thomas, F., Renaud, F. \& Poulin, R. 1998. Exploitation of manipulators: 'hitch-hiking' as a parasite transmission strategy. Anim. Behav. 56: 199-206.

Thompson, J.N. 1994. The Coevolutionary Process. University of Chicago Press, Chicago.

Trouvé, S., Sasal, P., Jourdane, J., Renaud, F. \& Morand, S. 1998. The evolution of life history traits in parasitic and freeliving platyhelminths: a new perspective. Oecologia 115: 370378.

Received 7 October 2003; revised 21 November 2003; accepted 23 November 2003 